\title{
Classification of EMG based Diseases using Fuzzy Logic at Second Level
}

\author{
Babita Pandey \\ Lovely Professional University \\ School of Computer Application \\ Phagwara, Punjab
}

\author{
Heena \\ Lovely Professional University \\ School of Computer Application \\ Phagwara, Punjab
}

\begin{abstract}
Intelligent diagnosis systems plays very important role in the medical field and particularly in diagnosis and classification of neuromuscular diseases (NMD). In such a field where uncertainty is always present, classical techniques of classification show a lack of efficiency thus leading to erroneous results. Fuzzy logic proved to be a suitable method for classification problems, particularly in NMDs. This paper aims to present a fuzzy logic based system for the classification of seven types of neuromuscular diseases at first level and classification of eight types of muscular dystrophy diseases at second level. The obtained results have shown good performance of the designed classifier.
\end{abstract}

\section{Keywords}

Intelligent diagnosis system, neuromuscular diseases, fuzzy logic, classification.

\section{INTRODUCTION}

Neuromuscular disease (NMD) is caused by muscular abnormality. These diseases are progressive in nature therefore their early diagnosis is very difficult. Some of the NMD are also not curable in the final stage. Therefore, we need a diagnostic method that accurately diagnoses the NMD in their early stage. Although a number of conventional and intelligent methods such as: heuristic methods, artificial neural networks (ANN), data mining (DM), case base reasoning (CBR), rule base reasoning (RBR) [1,2,3,9] are available for the diagnosis of NMD but they have their own limitations. Classical techniques of classification applied on bio-signal signals reveal inefficiency because of their badness of quality induced by the complex psychological and physiological state of the patients during recording besides the lack of accuracy of the used equipment [4]. Apart from this, the interpretation of bio signals by specialists is relatively hard and subjective because in some cases abnormalities cannot be distinguished easily due to ambiguity and blurriness. The problem of bad quality can be handled by the use of adequate noise filtering techniques [4].The problem of interference of classes can be solved through the integration of intelligent methods such as ANN-CBR. But still, the problem of uncertainty and imprecision of the knowledge in NMDs cannot be solved with classical or intelligent technique. Fuzzy logic proved to be a suitable method for solving such problems [6]. Apart from the above the problem is that most of the literature depicts the classification of NMD only in two or three classes [5,6]. The objective of this paper is to develop a fuzzy logic based system for the classification of seven types of neuromuscular diseases (NMD)at first level: muscular dystrophy (MD), ploymyositis (PO), endocrine myopathy (EM), metabolic myopathy (MM), neuropathy (N), poliomyelitis (PL) and myasthenia gravis (MG) using clinical parameters such as: physiological (muscle pain etc), cognitive (learning disability, hearing disability etc), motor parameters (difficulty in walking etc), psychological parameters (fatigue, anxiety etc.) and EMG parameters such as: amplitude, duration, phase etc and eight muscular dystrophy diseases at second level: duchenne muscular dystrophy(DMD),Becker muscular dystrophy(BMD), Congenital muscular dystrophy (CMD), Emery-Dreifuss muscular dystrophy(EDMD), Facioscapulohumeral muscular dystrophy (FSH), Limb-girdle muscular dystrophy (LGMD),myotonic muscular dystrophy (MMD)and Oculopharyngeal muscular dystrophy (OPMD).The rest of the paper is organized as follows. Section 2 covers the neuromuscular diseases and muscular dystrophy with their sign and symptoms. Section 3 describes the fuzzy logic method. Results are shown in Section 4. Finally, conclusions are drawn in section 5.

\section{NEUROMUSCULAR DISEASES : SIGN AND SYMPTOMS}

\subsection{First level disease}

Table 1 describes NMD diseases with their two important parameters: physio-psycho (PP) parameters and EMG signal characteristics (EMG). Physio-psycho parameter is further divided into three parts: is further divided into three parts: muscular physiology (M Phy)consisting of muscular (M) parameters such as: fibrillations $(\mathrm{FI})$, fasciculation $(\mathrm{Fa})$, muscle pain (MP), muscle inflammation (MI)and muscle stiffness(MS) (see columns 2-6 in Table 1); and motor activity (MA) parameters such as: difficulty in climbing stairs (CS), difficulty in walking (W), difficulty in running (R), difficulty in lifting above the head or shoulder (LH) and difficulty in rising above the chair(RC) [5] (see columns7-11 in Table 1), cognitive (C) and psychological (P) parameters. The cognitive parameters are visual disability (VD), touch disability (TC), speech disability (SD), hearing disability (HD) and learning disability (LD) (see columns 12-16 in Table 1). The psychological parameters are: fatigue (FT), anxiety(AN), depression (DE), isolation (IS) and frustration (FR) (see columns 17-21 in Table 1) (Kirmse,2008). The signal characteristics also divided into two parts: pattern characteristics such as: positive sharp wave (PSW), complex repetitive discharge (CRD) and mytonic discharge (MD) (see columns 22-24 in Table 1) and MUAP (motor unit action potential)parameters such as: amplitude (AM), duration (DU), phase (PH)and nerve conduction velocity (NVC) (see columns $25-28$ in Table1). In Table 1 first column contain the disease and columns 2-28contains the symptoms of the diseases. The columns 2-21 contain " $Y$ " if the respective symptom is present in the disease shown in the respective row. For example, Muscular Dystrophy(MD) has muscular symptoms such as: muscle pain, muscle in Flammarion and fibrillation. Therefore, the columns 2, 4 and 5contain "Y" as in Table 1. 
The other column of the Table 1 such as 22,23 and 24 of a particular row contains " $\mathrm{Y}$ " or " $\mathrm{N}$ " depending upon the characteristics of MUAP of the disease. The columns 25-27 contains" Y" if amplitude is less than $270 \mathrm{~lm}$, duration is greater than $10.2 \mathrm{~ms}$ and phase is greater than or equal to 5 , respectively , and " $\mathrm{N}$ " if amplitude is less than $160 \mathrm{~lm}$, duration is less than $9.8 \mathrm{~ms}$ and phase is less than 5, respectively. The column 28 contains " $Y$ " if there is normal decrease in nerve conduction velocity.

Table 1: Sign and Symptoms of NMD diseases at first level

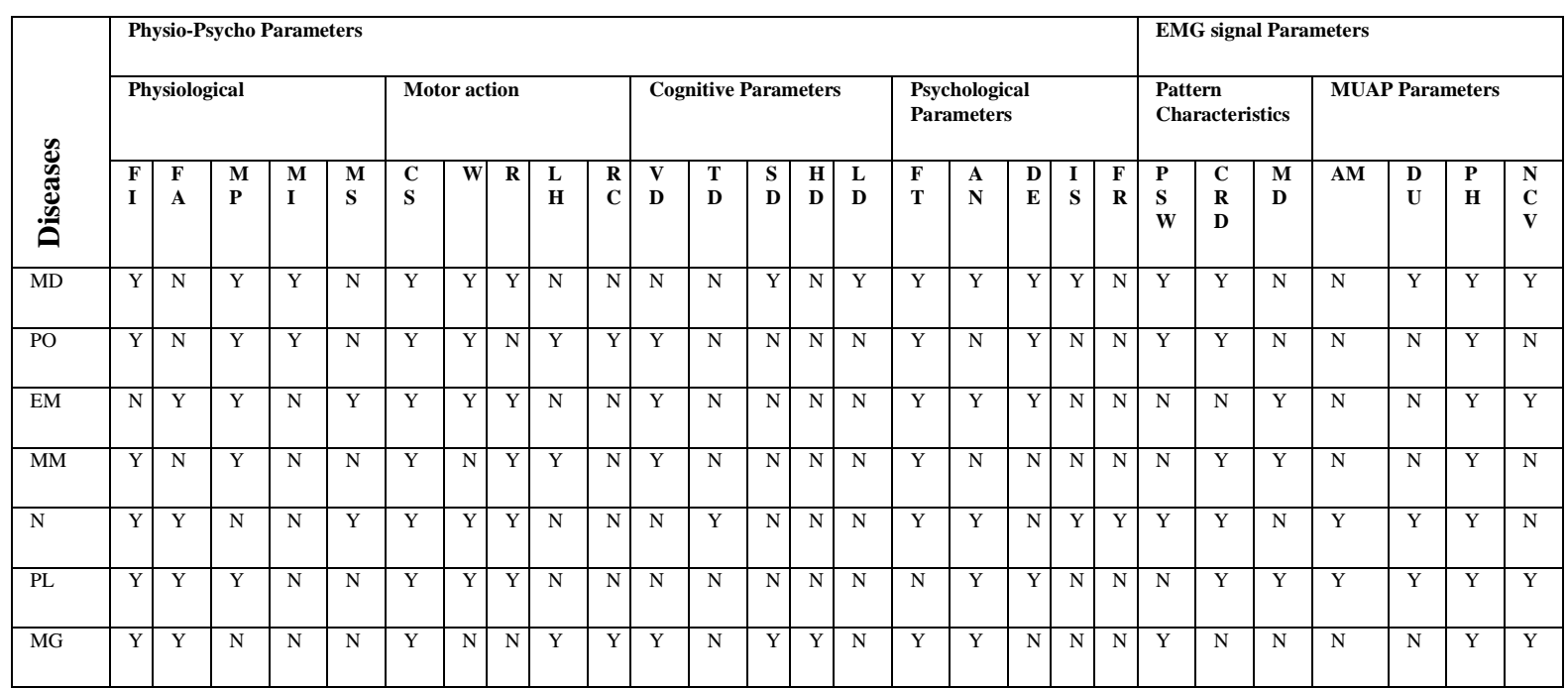

\subsection{Second level Disease}

Table 2 describes NMD diseases with their two important parameters: physio-psycho (PP) parameters and EMG signal characteristics (EMG). Physio-psycho parameter's further divided into four parts: muscular parameter which is further divided into two parts: Muscular(MUS) and motor(MOT) parameters such as: muscle pain (MP), muscle inflammation (MI)and muscle stiffness(MS)fibrillations(FI),fasciculation $(\mathrm{Fa})$, muscle pain (MP), muscle inflammation (MI)and muscle stiffness(MS) difficulty in lifting above the head or shoulder (LH) and difficulty in rising above the chair (RC) (see columns 2-10 in Table 2); The cognitive parameters are visual disability (VD), touch disability(TC), speech disability (SD), hearing disability (HD) and learning disability (LD) (see columns 12-15 in Table 2); The muscular psychological parameters are: fatigue (FT), anxiety (AN), depression (DP), isolation (IS), (see columns 16-21 in Table 2); The internal ligual parameters are (see columns $22-27$ in Table 2) lung problem(LP), Cardiac arrhythmia(CA), congestive heart
failure(CHF), chest pain(CP), heart muscle disease(HMD) and Skelton disorder(SKD). The EMG parameters are also divided into two parts: spontameous abnormality such as: positive sharp wave (PSW) and mytonic discharge (MD) (see columns 28-31 in Table 2) and MUAP (motor unit action potential)parameters such as: amplitude (AM), duration (DU), phase $(\mathrm{PH})$ and nerve conduction velocity (NVC) (see columns 32-35 in Table2). In Table 2first column contain the disease and columns 2-35 6contains the symptoms of the diseases. The columns 2-27 contain "Y" if the respective symptom is present in the disease shown in the respective row. For example, Duchenne Muscular Dystrophy (DMD) has muscular symptoms such as: muscle pain, muscle in Flammarion and fibrillation. Therefore, the columns 2, 4 and 5 contain "Y" as in Table 1. The other column of the Table 1 such as 22,23 and 24 of a particular row contains " $Y$ " or " $N$ " depending upon the characteristics of MUAP of the disease. 
Table2: Signs and symptoms of muscular dystrophy at second level.

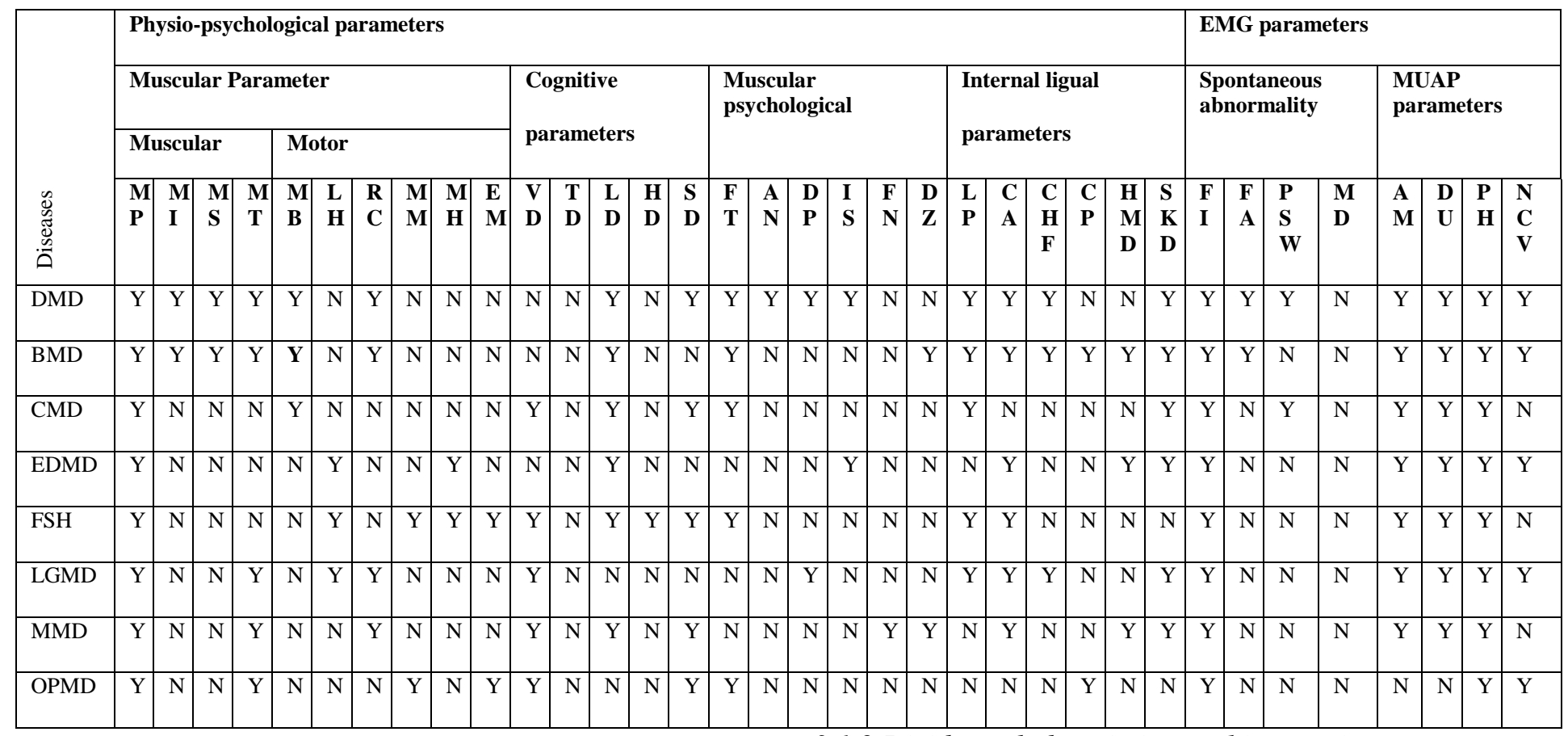

\section{FUZZY LOGIC}

The fuzzy model was elaborated based on experience and knowledge of neuromuscular diseases experts and literatures. These experts and literatures considered following inputs as important variables: physiological symptoms (Phy), motor symptoms (Mot), Cognitive symptoms (Cog), psychological symptoms (psy) as well as on EMG test variables such as: Spontaneous activity (SPO) and MUAP parameters (MUAP).

Fuzzy rule base system consists of four major components: (1) fuzzification module which translates crisp inputs into fuzzy values through linguistic variables, (2) fuzzy rule base; (3) fuzzy inference method; (4) defuzzification.

\subsection{Fuzzification}

In fuzzification step, numerical inputs and output variables are converted into linguistic terms or adjectives (such as low, high, big, small, etc.), and the corresponding degrees of the one or more several membership functions are determined. The fuzzification is done in following steps:

\subsubsection{Compute the domain interval of each input}

The domain interval of variable means that most probability the variables lie in their domain intervals. It is computed as follow:

a. Represent the six parameters Phy, Mot, Cog, Psy, SPO and MUAP given in table 1, into binary strings. Where number of bits are equivalent to number of sub parameters taken under particular parameters. For example, phychological parameter is represented by 5 bits.

b. Take the minimum and maximum decimal value represented by binary string. For example, using five bits the minimum and maximum decimal numbers represented by string are 0 and 31 . Therefore the domain interval for phy is $[0,31]$.

Similarly, the domain interval for Mot, Cog and Psy, SPO and MUAP are computed. The domain interval for Mot, Cog and Psy are $[0,31]$, for SPO is $[0,7]$ and for MUAP is $[0,15]$.

\subsubsection{Divide each domain interval into regions}

The next step in divide each domain interval into regions. The Physiological, Motor, Cognitive and Psychological symptom (Phy) are divided into five regions: very high (VH), high $(\mathrm{H})$, medium (M), low (L), very low (VL). The SPO and MUAP activities are divided into three regions: high $(\mathrm{H})$, medium (M), low (L). The corresponding fuzzy membership functions for input variables are shown in Figure 1. The range and fuzzy membership expression of all the input variables are given in Table 2. First column, in Table 1 contains the input parameters, $2^{\text {nd }}$ and $3^{\text {rd }}$ column contains the fuzzy set and their ranges. The $4^{\text {th }}$ column contains the fuzzy membership expression for the corresponding input parameter in $1^{\text {st }}$ column. The fuzzy output sets, is shown in Figure 2. The domain interval of output is computed as follow:

1. Determine the degree of given inputs Phy, Mot, Cog, Psy, SPO and MUAP in different regions. For this first convert the signs and symptoms given in Table 1, to its binary equivalent where $\mathrm{Y}$ is converted to ' 1 ' and $\mathrm{N}$ is converted to ' 0 '. For example, the binary string equivalent to phy in DMD is ' 10110 ' as shown in second cell of column 2 in Table 3 . Then convert the binary string to equivalent decimal number. The equivalent decimal to ' 10110 ' is 22 as shown in the right of equal sign in second cell of column 2 in Table 3 . This is called, score of phy for DMD. Similarly, the score of each input for each disease is calculated and shown in right side of equal to sign in Table 3. This score of Phy in DMD as from the Figure 1 has degree less than 0.5 in $\mathrm{VH}$, degree greater than 0.5 in $\mathrm{H}$ and zero degree in all other regions.

2. Assign a given inputs Phy, Mot, Cog, Psy, SPO and MUAP to maximum degree. For example, in case of DMD, Phy be in $\mathrm{H}$ set as shown in $2^{\text {nd }}$ cell of row headed by R1.For example, in case of DMD, Phy be in $\mathrm{H}$ set. Similarly, other are computed. Different levels are given to different set. The level of $\mathrm{VH}$ is $5, \mathrm{H}$ is $4, \mathrm{M}$ is $3, \mathrm{~L}$ is 2 and $\mathrm{VL}$ is 1 . 
3. Then, different weights are given to different inputs depending upon the number of bits used to represent the particular input. For example, the weight assigned to Phy ( $\mathrm{W}_{\text {phy }}$ )is 5 as 5 bit string is used to represent it. Similarly, the weight assigned to mot $\left(\mathrm{W}_{\text {mot }}\right), \operatorname{cog}\left(\mathrm{W}_{\text {cog }}\right)$, psy $\left(\mathrm{W}_{\mathrm{psy}}\right)$, spo $\left(\mathrm{W}_{\mathrm{spo}}\right)$, and $\operatorname{MUAP}\left(\mathrm{W}_{\text {muap }}\right)$, are 5, 5, 5, 5, 3 and 4 and .

4. Next step is to calculate the score of diseases as follows:

Score of DMD $=\mathrm{W}_{\text {phy }} *$ Level of Phy in DMD $+\mathrm{W}_{\text {mot }} *$ Level of Mot in DMD $+\mathrm{W}_{\mathrm{cog}}+$ Level of Cog in DMD+ $\mathrm{W}_{\mathrm{psy}} *$ Level of Psy in DMD $+\mathrm{W}_{\mathrm{spo}} *$

Table 3: Computation of score of diseases

\begin{tabular}{|l|l|l|l|l|l|l|l|}
\hline Diseases & Phy & Mot & Cog & Psy & SPO & MUAP & Score \\
\hline DMD & $10110=22$ & $11100=28$ & $00101=3$ & $11110=30$ & $110=6$ & $0111=7$ & 96 \\
\hline PO & $10110=22$ & $11011=27$ & $10000=16$ & $10100=20$ & $110=6$ & $0010=2$ & 97 \\
\hline EM & $01101=13$ & $11100=28$ & $10000=16$ & $11100=28$ & $001=1$ & $0011=3$ & 98 \\
\hline MM & $10100=6$ & $10110=22$ & $10000=16$ & $10000=16$ & $011=3$ & $0010=2$ & 77 \\
\hline N & $11001=25$ & $11100=28$ & $01000=8$ & $11011=27$ & $110=6$ & $1110=14$ & 110 \\
\hline PL & $11100=28$ & $11100=28$ & $00000=0$ & $01100=12$ & $011=3$ & $1111=15$ & 95 \\
\hline MG & $11000=24$ & $10011=19$ & $10110=22$ & $11000=24$ & $000=0$ & $0011=3$ & 108 \\
\hline
\end{tabular}

\subsubsection{Rule base}

The defined fuzzy rule base is formed by 7 rules (Table 4) and therefore is not complete because with six input features the number of combinations that can be made is greater than seven. That doesn't mean that the model is poorly
Level of SPO in DMD $+\mathrm{W}_{\text {muap }}+$ Level of MUAP in DMD

$=5 * 4+5 * 5+5 * 1+5 * 5+3$

$* 3+4 * 3=96$

Similarly, score of other diseases are calculated. The score of PO, EM, MM, N, PL and MG are 97, 98, 77, 110, 95 and 108 respectively in last column of Table 3 , the value given. These score of diseases decide the boundaries of output. The boundaries of output are [77, 110].The member ship functions used for input are trapezoidal and triangular functions. The membership function of output variable is shown in Figure 2.

\section{Table 4: Fuzzy Rule Base at first level}

\begin{tabular}{|l|l|l|l|l|l|l|l|}
\hline Rule & Phy & Mot & Cog & Psy & SPO & MUAP & Diagnosis \\
\hline R1 & H & VH & VL & VH & M & M & DMD \\
\hline R2 & H & VH & M & H & M & L & PO \\
\hline R3 & M & VH & M & VH & L & M & EM \\
\hline R4 & L & H & M & M & M & L & MM \\
\hline R5 & VH & VH & L & VH & M & H & N \\
\hline R6 & VH & VH & VL & M & M & H & PL \\
\hline R7 & VH & H & H & VH & L & M & MG \\
\hline
\end{tabular}



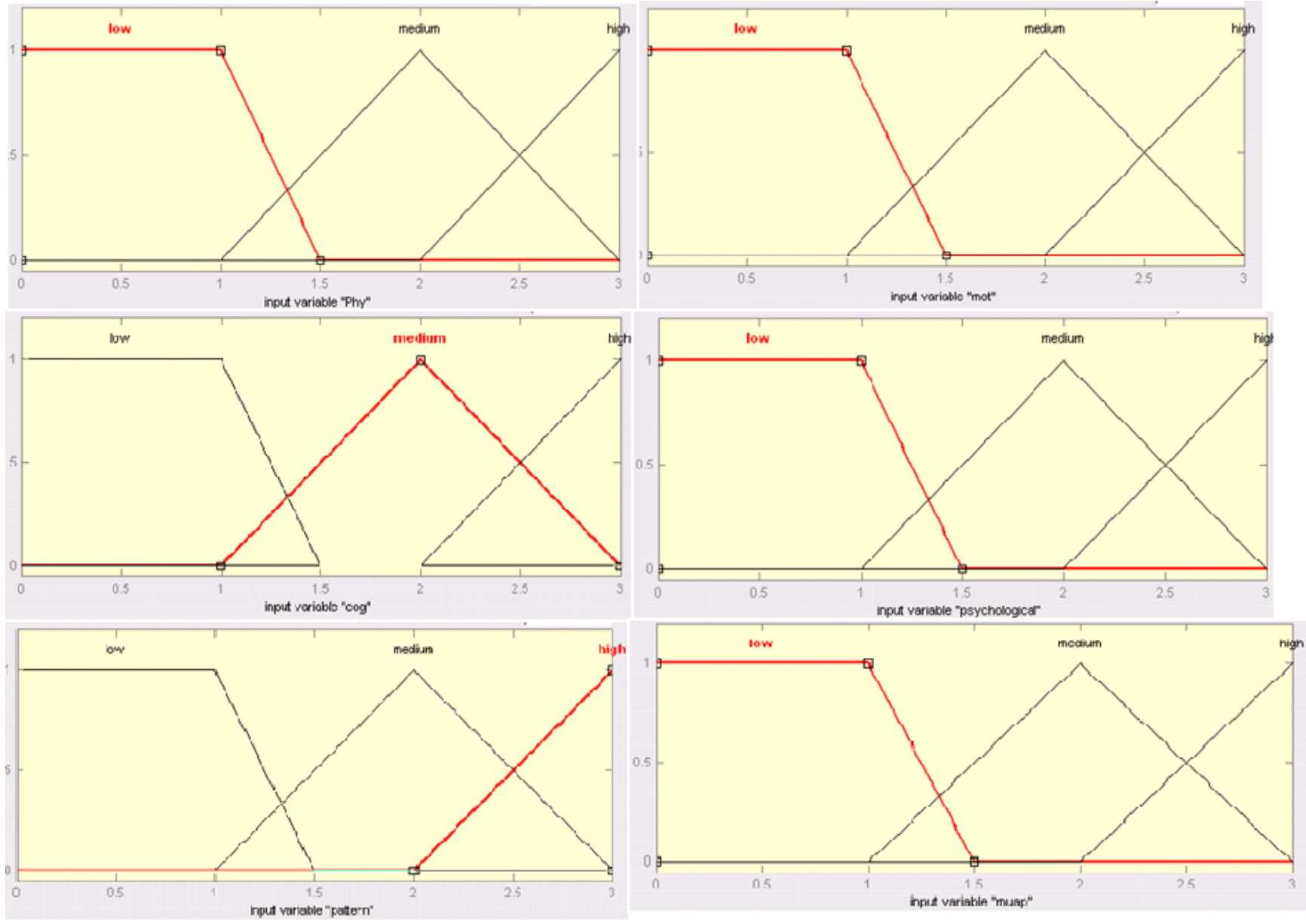

Figure 1: Membership function of input variables at First level

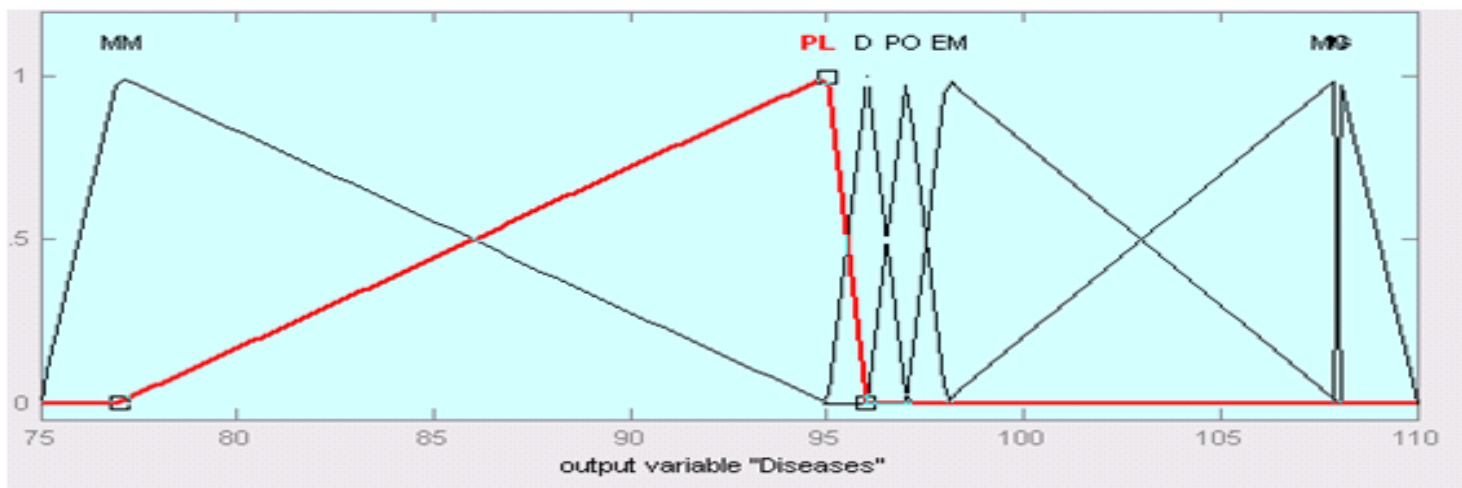

Figure 2: Membership function of output variables at first level.

\subsubsection{Fuzzy Inference and Defuzzification}

Designed system uses inference mechanism Mamdoni approach. The programming environment MATLAB 10a along with the FUZZY LOGIC TOOLBOX was used for the implementation of the fuzzy model and the classification algorithm. For defuzzification process, designed system uses "Centroid" method. On the basis of above-mentioned fuzzy steps, a detailed schematic of the MIMO (multiple inputs and multiple outputs) fuzzy system applied in this study is depicted.

\subsection{Fuzzy logic at second level}

At the second level the symptoms are : muscular symptoms (mus), motor symptoms (Mot), Cognitive symptoms (Cog), Muscular psychological symptoms(MusPsy) and Internal ligual (intlig) as well as on EMG test variables such as: Spontaneous abnormality (spo) and MUAP parameters (MUAP). Domain interval of output is calculated in second as it was calculated in the first level. 
Table 5: Computation of score of diseases at second level

\begin{tabular}{|l|l|l|l|l|l|l|l|l|}
\hline Diseases & mus & mot & cog & MusPsy & intlig & Spo & MUAP & Score \\
\hline DMD & $1111=15$ & $101000=40$ & $00101=5$ & $111100=57$ & $111001=57$ & $1110=14$ & $1111=15$ & 95 \\
\hline BMD & $1111=15$ & $101000=40$ & $00100=4$ & $100001=33$ & $111111=63$ & $1100=12$ & $1111=15$ & 85 \\
\hline CMD & $1000=8$ & $100000=32$ & $10101=21$ & $100000=32$ & $100001=33$ & $1010=10$ & $1110=14$ & 77 \\
\hline EDMD & $1000=8$ & $010010=18$ & $00100=4$ & $000100=4$ & $010011=19$ & $1000=8$ & $1111=15$ & 49 \\
\hline FSH & $1000=8$ & $010111=23$ & $10111=23$ & $100000=32$ & $110000=48$ & $1000=8$ & $1110=14$ & 83 \\
\hline LGMD & $1001=9$ & $011000=24$ & $10000=16$ & $001000=8$ & $111001=57$ & $1000=8$ & $1111=15$ & 66 \\
\hline MMD & $1001=9$ & $001000=5$ & $10101=21$ & $000011=3$ & $010011=19$ & $1000=8$ & $1110=14$ & 53 \\
\hline OPMD & $1001=9$ & $000101=5$ & $10001=17$ & $100000=32$ & $000100=4$ & $1000=8$ & $0011=3$ & 51 \\
\hline
\end{tabular}

Fuzzy Rules at second level

Fuzzy rules are generated with the help of computed scores.

Table 6: fuzzy rules at second level

\begin{tabular}{|c|c|c|c|c|c|c|c|c|}
\hline RULE & Mus & Mot & $\operatorname{Cog}$ & MusPsy & Intlng & SPO & MUAP & Diseases \\
\hline R1 & $\mathrm{H}$ & $\mathrm{H}$ & $\mathrm{L}$ & $\mathrm{H}$ & $\mathrm{H}$ & $\mathrm{H}$ & $\mathrm{H}$ & DMD \\
\hline R2 & $\mathrm{H}$ & $\mathrm{H}$ & $\mathrm{L}$ & $\mathrm{M}$ & $\mathrm{H}$ & $\mathrm{H}$ & $M$ & BMD \\
\hline R3 & $\mathrm{L}$ & $\mathrm{H}$ & $\mathrm{H}$ & $\mathrm{M}$ & $\mathrm{M}$ & $\mathrm{H}$ & $\mathrm{L}$ & CMD \\
\hline $\mathrm{R} 4$ & $\mathrm{~L}$ & $\mathrm{M}$ & $\mathrm{L}$ & $\mathrm{L}$ & $\mathrm{L}$ & $\mathrm{H}$ & $\mathrm{L}$ & EDMD \\
\hline R5 & $\mathrm{L}$ & $\mathrm{L}$ & $\mathrm{H}$ & $\mathrm{M}$ & $\mathrm{H}$ & $\mathrm{H}$ & $\mathrm{L}$ & FSH \\
\hline R6 & $\mathrm{L}$ & $\mathrm{M}$ & $\mathrm{M}$ & $\mathrm{L}$ & $\mathrm{H}$ & $\mathrm{H}$ & $\mathrm{L}$ & LGMD \\
\hline R7 & $\mathrm{L}$ & $\mathrm{L}$ & $\mathrm{H}$ & $\mathrm{L}$ & $\mathrm{L}$ & $\mathrm{H}$ & $\mathrm{L}$ & MMD \\
\hline R8 & $\mathrm{L}$ & $\mathrm{L}$ & $\mathrm{H}$ & $\mathrm{M}$ & $\mathrm{L}$ & $\mathrm{L}$ & $\mathrm{L}$ & OPMD \\
\hline R9 & $\mathrm{H}$ & $\mathrm{M}$ & $\mathrm{L}$ & $\mathrm{H}$ & $\mathrm{H}$ & $\mathrm{H}$ & $\mathrm{H}$ & BMD \\
\hline R10 & $\mathrm{H}$ & $\mathrm{H}$ & $\mathrm{H}$ & $\mathrm{H}$ & $\mathrm{H}$ & $\mathrm{H}$ & $\mathrm{H}$ & DMD \\
\hline R11 & $\mathrm{H}$ & $\mathrm{H}$ & $\mathrm{L}$ & $\mathrm{H}$ & $\mathrm{M}$ & $\mathrm{H}$ & $\mathrm{H}$ & BMD \\
\hline R12 & $\mathrm{H}$ & $\mathrm{H}$ & $\mathrm{M}$ & $\mathrm{M}$ & $\mathrm{H}$ & $\mathrm{H}$ & $\mathrm{M}$ & BMD \\
\hline R13 & $\mathrm{M}$ & $\mathrm{H}$ & $\mathrm{L}$ & $\mathrm{M}$ & $\mathrm{H}$ & $\mathrm{H}$ & $\mathrm{M}$ & FSH \\
\hline R14 & $\mathrm{H}$ & $\mathrm{H}$ & $\mathrm{L}$ & $\mathrm{M}$ & $\mathrm{H}$ & $\mathrm{H}$ & $\mathrm{L}$ & FSH \\
\hline R15 & $\mathrm{L}$ & $\mathrm{M}$ & $\mathrm{H}$ & $\mathrm{M}$ & $\mathrm{M}$ & $\mathrm{H}$ & $\mathrm{L}$ & CMD \\
\hline R16 & $\mathrm{L}$ & $\mathrm{H}$ & $\mathrm{H}$ & $\mathrm{L}$ & $\mathrm{M}$ & $\mathrm{H}$ & $\mathrm{L}$ & CMD \\
\hline R17 & $\mathrm{L}$ & $\mathrm{H}$ & $\mathrm{H}$ & $\mathrm{M}$ & $\mathrm{M}$ & $\mathrm{M}$ & $\mathrm{L}$ & CMD \\
\hline R18 & $\mathrm{L}$ & $\mathrm{M}$ & $\mathrm{L}$ & $\mathrm{M}$ & $\mathrm{L}$ & $\mathrm{H}$ & $\mathrm{L}$ & MMD \\
\hline R19 & $\mathrm{L}$ & $\mathrm{M}$ & $\mathrm{L}$ & $\mathrm{L}$ & $\mathrm{L}$ & $\mathrm{M}$ & $\mathrm{L}$ & EDMD \\
\hline R20 & $\mathrm{L}$ & $\mathrm{H}$ & $\mathrm{L}$ & $\mathrm{L}$ & $\mathrm{L}$ & $\mathrm{H}$ & $\mathrm{L}$ & MMD \\
\hline R21 & $\mathrm{L}$ & $\mathrm{M}$ & $\mathrm{H}$ & $\mathrm{M}$ & $\mathrm{H}$ & $\mathrm{H}$ & $\mathrm{L}$ & CMD \\
\hline R22 & $\mathrm{L}$ & $\mathrm{L}$ & $\mathrm{H}$ & $\mathrm{L}$ & $\mathrm{H}$ & $\mathrm{H}$ & $\mathrm{L}$ & LGMD \\
\hline R23 & $\mathrm{L}$ & $\mathrm{L}$ & $\mathrm{H}$ & $\mathrm{M}$ & $\mathrm{H}$ & $\mathrm{M}$ & $\mathrm{L}$ & LGMD \\
\hline R24 & $\mathrm{L}$ & $\mathrm{M}$ & $\mathrm{M}$ & $\mathrm{L}$ & $\mathrm{H}$ & $\mathrm{H}$ & $M$ & LGMD \\
\hline R25 & $\mathrm{L}$ & $\mathrm{H}$ & $\mathrm{M}$ & $\mathrm{L}$ & $\mathrm{H}$ & $\mathrm{H}$ & $\mathrm{L}$ & CMD \\
\hline R26 & $\mathrm{L}$ & $\mathrm{M}$ & $\mathrm{M}$ & $\mathrm{M}$ & $\mathrm{H}$ & $\mathrm{H}$ & $\mathrm{L}$ & LGMD \\
\hline R27 & $\mathrm{L}$ & $\mathrm{M}$ & $\mathrm{H}$ & $\mathrm{L}$ & $\mathrm{L}$ & $\mathrm{H}$ & $\mathrm{L}$ & MMD \\
\hline R28 & $\mathrm{L}$ & $\mathrm{L}$ & $\mathrm{H}$ & $\mathrm{L}$ & $\mathrm{L}$ & $\mathrm{M}$ & $\mathrm{L}$ & EDMD \\
\hline R29 & M & $\mathrm{L}$ & $\mathrm{H}$ & $\mathrm{L}$ & $\mathrm{L}$ & $\mathrm{H}$ & $\mathrm{L}$ & MMD \\
\hline R30 & $\mathrm{L}$ & M & $\mathrm{H}$ & M & $\mathrm{L}$ & $\mathrm{L}$ & $\mathrm{L}$ & MMD \\
\hline R31 & $\mathrm{L}$ & $\mathrm{L}$ & $\mathrm{M}$ & $\mathrm{M}$ & $\mathrm{L}$ & $\mathrm{L}$ & $\mathrm{L}$ & EDMD \\
\hline R32 & $\mathrm{L}$ & $\mathrm{L}$ & $\mathrm{H}$ & $\mathrm{M}$ & $\mathrm{L}$ & $\mathrm{M}$ & $\mathrm{L}$ & MMD \\
\hline R33 & $\mathrm{H}$ & $\mathrm{H}$ & $M$ & $\mathrm{M}$ & $\mathrm{H}$ & $\mathrm{H}$ & $\mathrm{H}$ & DMD \\
\hline R34 & $\mathrm{H}$ & $\mathrm{H}$ & $\mathrm{M}$ & $\mathrm{H}$ & $\mathrm{H}$ & $\mathrm{H}$ & $\mathrm{H}$ & DMD \\
\hline
\end{tabular}




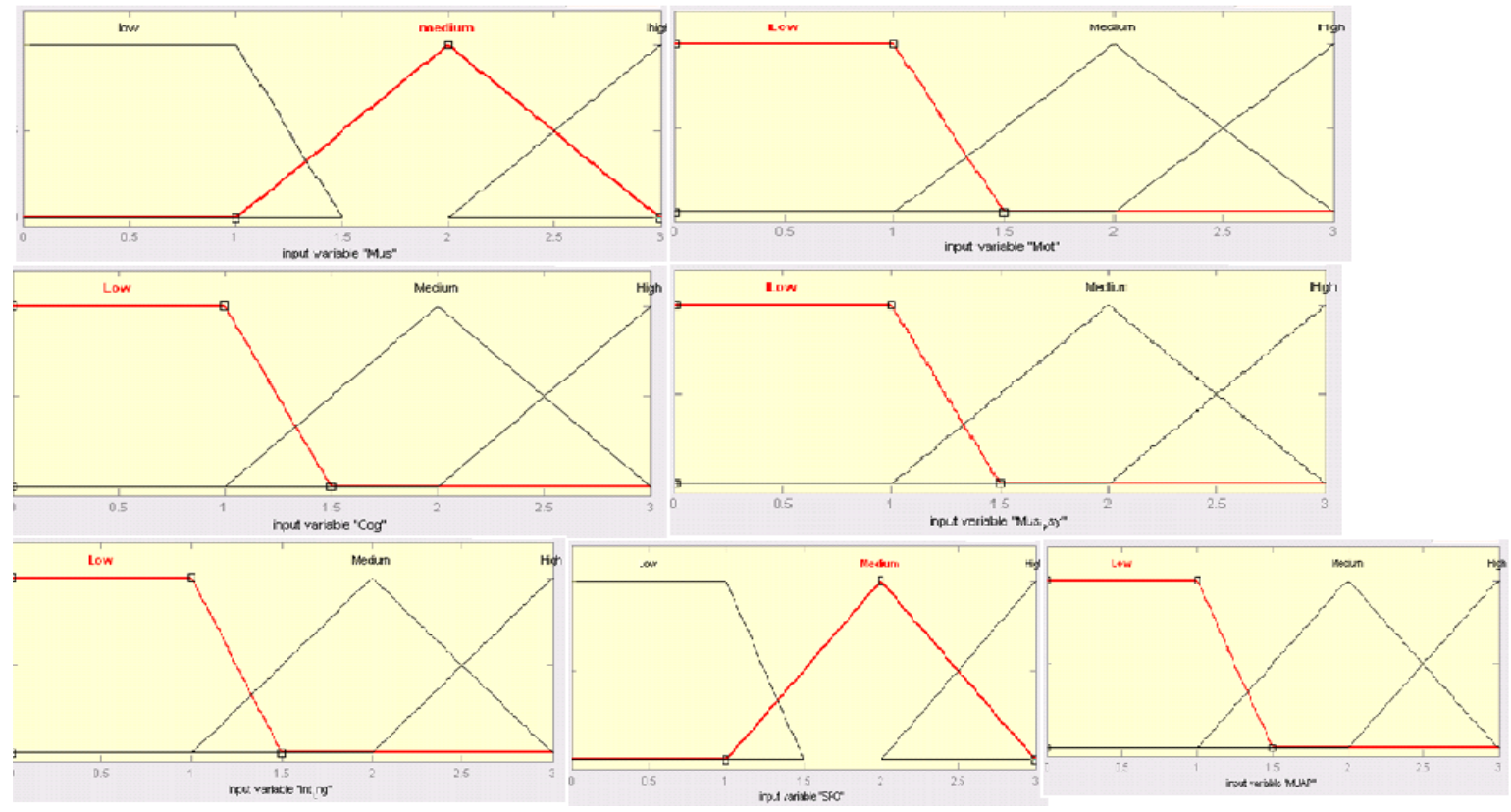

Figure 3: Membership functions of input variables at second level

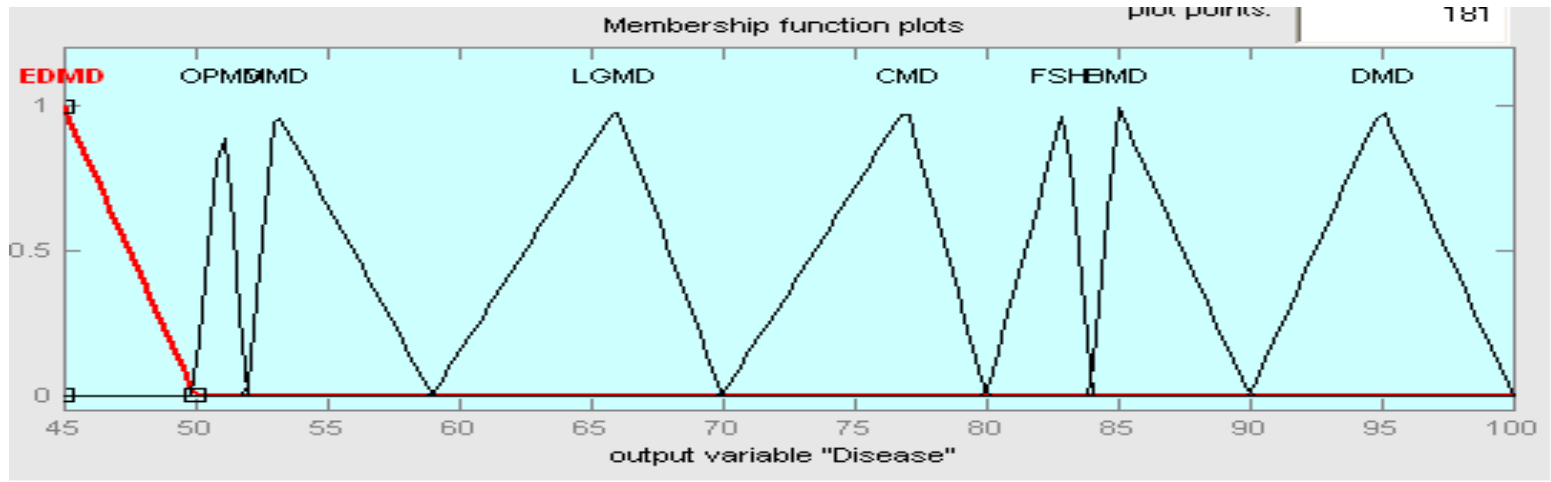

Figure4: Membership functions of output variables at second level

\section{RESULT}

\subsection{Result at first level diagnosis of EMG \\ based diseases}

The test data consist of 61 cases. It was created from expert opinion and literature survey. It consists of 10 DMD cases, 20 PO cases, 5 EM cases, $6 \mathrm{MM}$ cases, $5 \mathrm{~N}$ cases, $5 \mathrm{PL}$ cases and $10 \mathrm{MG}$ cases. The accuracy of a classifier can be computed using sensitivity and specificity. For the given seven classes, we consider in terms of positive tuples (diagnosis =any particular disease like DMD) versus negative tuples (eg., diagnosis $=$ not that particular disease like not DMD). True positives refer to the positive tuples that were correctly labeled by the classifier, while true negatives are the negative tuples that were correctly labeled by the classifier. False positives are the negative tuples that were incorrectly labeled by the classifier, while false negatives are the positive tuples that were incorrectly labeled by the classifier. These measures are defined as

\section{Sensitivity $=\operatorname{tpos} / \mathrm{pos}$}

tpos is the number of true positives (i.e. samples that were correctly classified suffering with a particular disease) and pos is the number of positive (i.e. sample that are actually suffering with a particular disease) samples. For example, while testing among the 10 cases of DMD, 8 are classified correctly by fuzzy logic and 2 are classified wrong. Therefore, Sensitivity $==8 / 10=0.8$.

Specificity $=$ tneg/ neg

tneg is the number of true negatives (i.e. samples that were not suffering with particular disease and were correctly classified) an neg is the number of positive (i.e. samples that were actually not suffering with particular disease) samples and fpos is the number of false positives ("samples that are actually not suffering with a particular disease but were incorrectly classified). For example, while testing among the 51 cases that are not suffering with DMD, 47 are classified correctly by fuzzy logic and 4 are classified wrong. Therefore, Sensitivity $=47 / 51=0.92$.

The true positives, true negatives, false positives and false negatives are also useful in assessing the costs and benefits (or risks and gains) associated with a classification model. 
Table7: Comparative table of Measures of different disease

\begin{tabular}{|l|l|l|l|l|l|l|l|}
\hline Measure & DMD & PO & EM & MM & N & PL & MG \\
\hline Specificity & 0.92 & 0.9 & 0.98 & 0.96 & 0.98 & 0.98 & 0.92 \\
\hline Sensitivity & 0.8 & 0.9 & 0.8 & 0.66 & 0.8 & 0.8 & 0.8 \\
\hline
\end{tabular}

The results are shown in Table 7, in terms of sensitivity and specificity. The results indicate that the proposed method performs well in classifying NMD's. Plots of the seven results given in Table 7 are shown in ROC space in the Figure 5. For all the diseases, the result is above the diagonal, which represent good classification results.

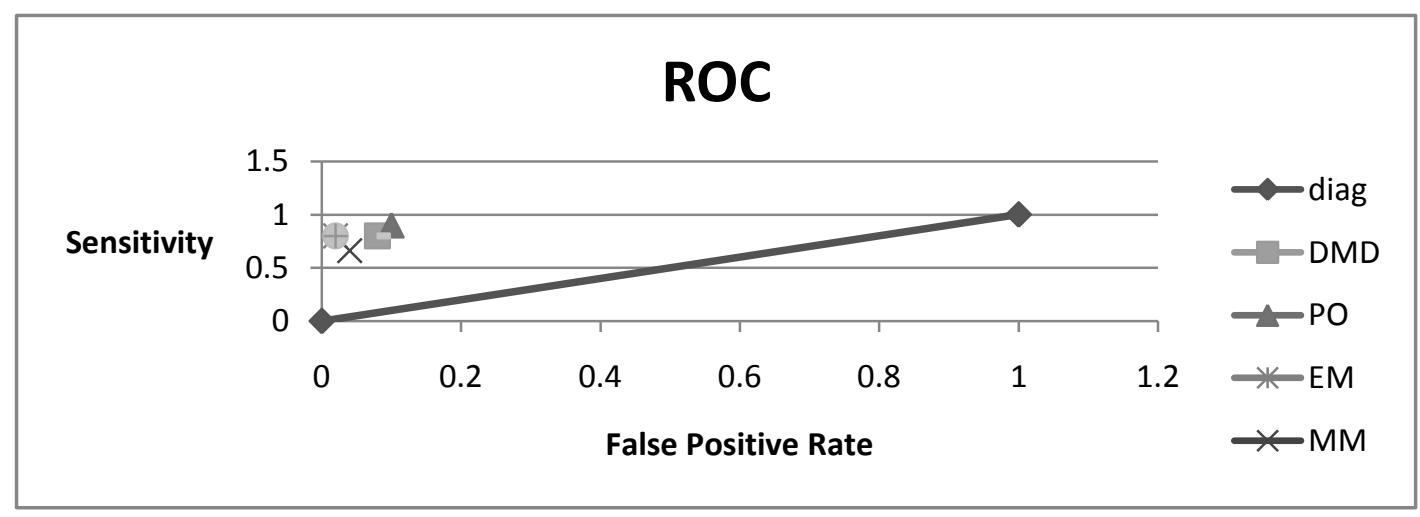

\subsection{Result at second level diagnosis of EMG based diseases:}

The test data consist of 34 cases. It was created from expert opinion and literature survey. It consists of 4 DMD cases, 4 BMD cases, 6CMD cases, 4 EDMD cases, 3 FSH cases, 5

LGMD cases and 7 MMD cases and 1 OPMD case. The sensitivity and specificity for each disease are computed and shown in table 8 .

Table 8: specificity and sensitivity of diseases at second level.

\begin{tabular}{|l|l|l|l|l|l|l|l|l|}
\hline Measure & DMD & BMD & CMD & EDMD & FSH & LGMD & MMD & OPMD \\
\hline Specificity & 0 & 0 & 0.94 & 0 & 0 & 0 & 0.88 & 0 \\
\hline Sensitivity & 1 & 1 & 0.06 & 1 & 1 & 1 & 0.71 & 1 \\
\hline
\end{tabular}

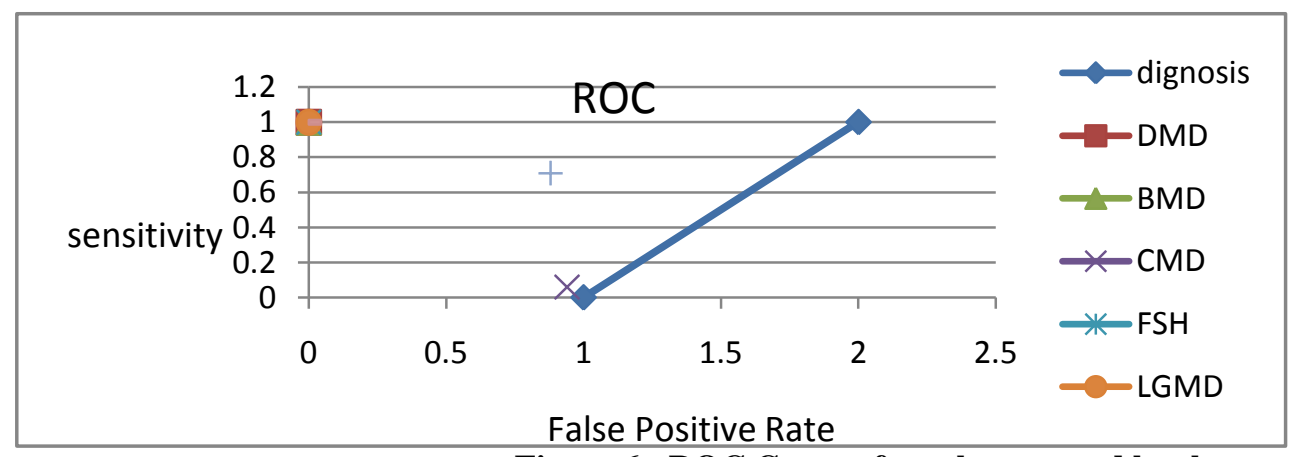

Figure 6: ROC Curve of result at second level

\section{CONCLUSION}

This work integrates in the context of medical system development. It has been devoted particularly to classify NMDs based at first level on the analysis of the EMG signal such as: amplitude, phase and duration and clinical parameters such as: physical, cognitive, psychological and motor parameters using fuzzy logic, and at second level analysis of muscular dystrophy diseases using signal such as muscular, cognitive, Muscular psychological, Internal ligual, Spontaneous abnormality and MUAP parameters using fuzzy logic.

After presenting the limits of the classical approach and intelligent technique such as ANN, CBR and RBR,I n solving the problem of classification in the medical domain, we have seen the reasons for choosing a new fuzzy method to achieve the goal of designing a system capable of making reliable diagnoses. Finally, the classification results were presented.

The results were satisfactory and were shown in the form of sensitivity and specificity. However, the design of the fuzzy model, including the fuzzy rule base and the membership functions, was rather hard to achieve Although it may seem simple. This difficulty was due to then tuitive nature of the human way of thinking which cannot be easily transformed to a numerical model. 


\section{REFERENCES}

[1] R. B. Mishra, R. S. Rao, A heuristic pattern detection algorithm for assessment of EMG signals, Indian Journal of the Institution of Engineers 76 (1997) 52-58.

[2] B. Pandey, R.B.Mishra, Case-based reasoning and data mining integrated method for the diagnosis of some neuromuscular disease, Int. J. Medical Engineering and Informatics 3(1) (2011) 1-15.

[3] B. Pandey, R.B. Mishra, An integrated intelligent computing model for the interpretation of EMG based neuromuscular diseases, Expert System with Application 36 (5) (2009) 9201-9213.

[4] A. MESSAOUD, M. B. MESSAOUD, A. KACHOURI and F. SELLAMI, Fuzzy logic based system for classification of atrial fibrillation cardiac arrhythmias, IEEE, 2006, pp. 347-350

[5] A. Subasi, Medical decision support system for diagnosis of neuromuscular disorders using DWT and fuzzy support vector machines, Computers in Biology and Medicine archive Volume 42 Issue 8, August, 2012,Pages 806-815.

[6] A. P. Picon, R. S. Ortega, R. Watari, C. Sartor, I. C. N. Sacco, Classifiction of diabetic neuropathy: a new approach taking uncertainity into account using fuzzy logic, Clinics, 2012, 67(2):151-156.

[7] B. Pandey, R.B. Mishra, Data Mining Approaches for the EMG absed classification of neuromuscular diseases, National conference on Artificial Intelligence and Agents(2011),136-148.

[8] B.Pandey, R. B. Mishra,Performance index assessment of intelligent computing methods in EMG-based neuromuscular diseases.IJKESDP, 4(1):42-71(2013).

[9] B.Pandey, R. B. Mishra , An Intelligent Method Model For two level diagnoses of Neuromuscular Diseases,IJKESDP, (2014),(accepted) 